\title{
A NEW GEOMETRIC ALGORITHM TO GENERATE SMOOTH INTERPOLATING CURVES ON RIEMANNIAN MANIFOLDS
}

\author{
RUI C. RODRIGUES, F. SILVA LEITE AND JANUSZ JAKUBIAK
}

\begin{abstract}
This paper presents a new geometric algorithm to construct a $C^{k}$ smooth spline curve that interpolates a given set of data (points and velocities) on a complete Riemannian manifold. Although based on a modification of the De Casteljau procedure, this new algorithm is implemented in only three steps, independently of the required degree of smoothness, and therefore introduces a significant reduction in complexity. The key role is played by the choice of an appropriate smoothing function, which is defined as soon as the degree of smoothness is fixed.
\end{abstract}

\section{Introduction}

We propose a new algorithm, which has a pure geometric interpretation, in order to address the following interpolation problem.

Generate a $C^{k}$-smooth $(k \geqslant 1)$ curve $s:[a, b] \subset \mathbb{R} \longrightarrow \mathrm{M}$, on a complete Riemannian manifold $\mathrm{M}$, which fulfills a set of interpolation conditions of the form

$$
s\left(t_{i}\right)=p_{i} \quad \text { and } \quad \dot{s}\left(t_{i}\right)=v_{i},
$$

for a given partition $\Delta: a=t_{0}<t_{1}<\cdots<t_{m}=b$ of the time interval $[a, b]$, given points $p_{i}$ on $\mathrm{M}$ and vectors $v_{i}$ tangent to $\mathrm{M}$ at $p_{i}, i=0,1, \ldots, m$.

Many solutions and some efficient algorithms have been proposed to solve similar interpolation problems (where points and velocities are prescribed); these were motivated by applications in many areas of engineering, like motion-planning problems in robotics or object animation, which is required in computer graphics. In most cases, $\mathbf{M}$ is simply a Lie group or a sphere.

One development was achieved by Shoemake [18], introducing the idea of using quaternions to solve interpolation problems such as the one above, in the sphere $S^{3}$. This work was mainly motivated by applications in computer animation, and the approach may be generalized to higher-dimensional spheres.

The De Casteljau algorithm [5] is a well-known algorithm to generate polynomial curves in Euclidean spaces, based on recursive linear interpolation, which can be used to solve the proposed interpolation problem when the required degree of smoothness is equal to one and the manifold is $\mathbb{R}^{n}$. See Farin [6] for a general presentation of the De Casteljau method.

All the authors were supported by project POSI/SRI/41618/2001 and ISR-Coimbra.

The first author was also supported by a PRODEP grant, and the third author was also supported by Control Training Site HPMT-CT-2001-00278.

Received 26 April 2005, revised 10 June 2005; published 5 December 2005.

2000 Mathematics Subject Classification 41A15, 53A04, 53C22, 65D07

(C) 2005, Rui C. Rodrigues, F. Silva Leite and Janusz Jakubiak 
Other alternatives for Euclidean spaces, proposed by Nagy and Vendel [10] and Rodrigues, Silva Leite and Rosa [15], are based on convex combinations of rather simple curves, like line segments and circular arcs.

There are a number of references to work dealing with Bezier/De Casteljau algorithms on the manifolds $\mathrm{SO}(3), S^{2}, S^{3}$ and $\mathrm{SE}(3)$. In addition to the work by Shoemake [18], we also mention Barr, Currin, Gabriel and Hughes [1], Chen [2], Ge and Ravani [7], Kim, Kim and Shin [9], Nielson [11] and Nielson and Heiland [12]. The objective in most of these papers is to do interpolation on $\mathrm{SO}(3)$ using the fact that rotations in $\mathbb{R}^{3}$ may be represented by unit quaternions. This approach does not generalize to higher-dimensional manifolds. In this paper, we develop a general method for $m$-dimensional spheres that also includes the particular case of unit quaternions.

Extensions of De Casteljau's algorithm to Riemannian manifolds, Lie groups and spheres can be found in the work of Crouch, Kun and Silva Leite [3, 4] and Park and Ravani [13]. The idea common to such generalizations is the replacement of linear interpolation by geodesic interpolation. In Jakubiak, Silva Leite and Rodrigues [8], linear interpolation techniques have been replaced by polynomial interpolation, in order to improve the complexity of the De Casteljau algorithm.

The algorithm that we are about to present is performed in only three steps, no matter what degree of smoothness is required. It is based on a modification of the De Casteljau construction of a $C^{1}$-smooth cubic spline, and uses some ideas from the recent work already mentioned. We start with a review of the De Casteljau construction in Section 2. Another important feature, which is also shared by the De Casteljau procedure, relies on the fact that the calculation of each spline segment depends only on the local data. This is particularly useful in applications, since any change in the data at a particular instant of time requires only the re-calculation of two segments of the spline. This is not the case for some classical interpolating spline schemes, for which a single change in the data will mean an entire re-calculation of the spline curve.

The new geometric algorithm is first presented and discussed for Euclidean spaces. This will help with the visualization of its main features, and will motivate its generalization to other complete Riemannian manifolds. However, the algorithm is useful as a computational device only when the explicit implementation details of the algorithm have been worked out. In general, this objective is not reachable, but for some specific cases, like connected and compact Lie groups or spheres, we are able to calculate the interpolating curves in closed form and derive expressions for their derivatives, in order to be able to check the degree of smoothness at the interpolating points.

This paper is the natural evolution and an extended version of the paper by Rodrigues and Silva Leite [14], presented in the minisymposium 'Geometric optimization with applications in numerical linear algebra, robotics, and computer vision' at the MTNS2004 Conference.

\section{The De Casteljau algorithm revisited}

The De Casteljau algorithm is a geometric algorithm, and is one of the best-known algorithms used to generate polynomial spline curves in general Euclidean spaces. Its importance also follows from the simple geometric construction that is performed, which is based on the application of successive linear interpolations.

The classical De Casteljau algorithm is used to construct parameterized polynomial curves, of any given degree $d$, joining two points in $\mathbb{R}^{n}$. A sequence of $d-1$ points in $\mathbb{R}^{n}$ 
is used to implement the algorithm, and for that reason they are called control points; see Farin [6] for details.

We now show how the De Casteljau algorithm can be used to generate a curve in $\mathbb{R}^{n}$ that fulfills all the interpolation conditions (1).

For instance, from the given points $p_{0}, p_{1}$ and the given vectors $v_{0}, v_{1}$, two control points, namely $x_{0}$ and $x_{1}$, are uniquely determined by

$$
x_{0}=p_{0}+\frac{1}{3} v_{0} \quad \text { and } \quad x_{1}=p_{1}-\frac{1}{3} v_{1} .
$$

In this case the classical De Casteljau algorithm is performed in three steps, and the resulting curve will be a cubic polynomial. For simplicity, we may consider the time interval $[0,1]$ instead of $\left[t_{0}, t_{1}\right]$.

In the first step, the following three line segments are computed:

$$
\alpha_{0}(t)=p_{0}+t\left(x_{0}-p_{0}\right) ; \quad \alpha_{1}(t)=x_{0}+t\left(x_{1}-x_{0}\right) ; \quad \alpha_{2}(t)=x_{1}+t\left(p_{1}-x_{1}\right) .
$$

Then, two new curves are generated using the curves constructed in the previous step:

$$
\beta_{0}(t)=(1-t) \alpha_{0}(t)+t \alpha_{1}(t) ; \quad \beta_{1}(t)=(1-t) \alpha_{1}(t)+t \alpha_{2}(t)
$$

REMARK 2.1. We can say that $\beta_{0}$ and $\beta_{1}$ are generated from a convex combination of the curves $\alpha_{0}, \alpha_{1}$ and $\alpha_{1}, \alpha_{2}$ respectively.

These two curves are finally combined in a similar way to generate the cubic polynomial curve given by

$$
\begin{aligned}
s_{0}(t) & =(1-t) \beta_{0}(t)+t \beta_{1}(t) \\
& =(1-t)^{2} \alpha_{0}(t)+2 t(1-t) \alpha_{1}(t)+t^{2} \alpha_{2}(t) .
\end{aligned}
$$

If the classical De Casteljau construction is repeated for each other interval $\left[t_{i}, t_{i+1}\right]$, one obtains a spline curve $t \longmapsto s(t)$ in $\mathbb{R}^{n}$ which is the result of the concatenation of all polynomial segments $t \longmapsto s_{i}(t)$ and has the following final form:

$$
s(t)=s_{i}\left(\frac{t-t_{i}}{t_{i+1}-t_{i}}\right), \quad t \in\left[t_{i}, t_{i+1}\right], i=0,1 \ldots, m-1 .
$$

The spline curve $t \longmapsto s(t)$ is locally a cubic polynomial and satisfies the interpolation conditions (1), but it is only $C^{1}$-smooth (at each instant $t_{i}$ ).

To generate a $C^{k}$-smooth curve using the De Casteljau algorithm, one needs to prescribe $k$ derivatives at each instant $t_{i}$. The construction of each spline segment $t \longmapsto s_{i}(t)$ will require $2 k$ control points and the computation of $(2 k+1)(k+1)$ curves, performed in $2 k+1$ steps. It is clear that the computational cost of this algorithm increases substantially with $k$.

The De Casteljau algorithm has been generalized to complete Riemannian manifolds $[4,13]$, and this was mainly due to the fact that the algorithm is geometrically based. The idea is quite simple. The linear interpolation procedure in the classical case is simply replaced by geodesic interpolation. When applied to interpolation problems, the resulting curve has the same degree of smoothness as in the Euclidean case, but the implementation of the algorithm is much harder, even for low-dimensional cases. 


\section{A new geometric algorithm in $\mathbb{R}^{n}$}

We first consider the case when $M$ is $\mathbb{R}^{n}$ equipped with the Euclidean metric. The geometric algorithm proposed here is based on a modification of the De Casteljau algorithm, but has the ability to generate a spline curve in only three steps, with any required degree of smoothness. This property of our algorithm is due to the role played by a smoothing function $\phi$.

We now show how to compute the curve $t \longmapsto s_{i}(t) \in \mathbb{R}^{n}$, which connects the point $p_{i}$ at $t=0$ to $p_{i+1}$ at $t=1$, with initial and final velocities $v_{i}$ and $v_{i+1}$ (again, we use [0,1] instead of $\left.\left[t_{i}, t_{i+1}\right]\right)$. As in the De Casteljau algorithm, we use two control points, which are now

$$
x_{i}=p_{i}+v_{i}, \quad x_{i+1}=p_{i+1}-v_{i+1},
$$

and three steps. First, we define three line segments:

$$
\underline{\text { Step } 1}\left\{\begin{array}{l}
l_{i}(t)=p_{i}+t\left(x_{i}-p_{i}\right)=p_{i}+t v_{i}, \\
c_{i}(t)=p_{i}+t\left(p_{i+1}-p_{i}\right), \\
r_{i}(t)=x_{i+1}+t\left(p_{i+1}-x_{i+1}\right)=p_{i+1}+(t-1) v_{i+1},
\end{array}\right.
$$

which will play the roles of left, center and right components of the spline segment $t \longmapsto s_{i}(t)$. Notice that the left and right components satisfy

$$
\begin{array}{ll}
l_{i}(0)=p_{i}, & r_{i}(1)=p_{i+1}, \\
l_{i}(0)=v_{i}, & \dot{r}_{i}(1)=v_{i+1}, \\
l_{i}^{(j)}(0)=0, & r_{i}^{(j)}(1)=0, \quad j \geqslant 2 .
\end{array}
$$

REMARK 3.1. The three components are such that $c_{i}(0)=l_{i}(0)=p_{i}$ and $c_{i}(1)=r_{i}(1)=$ $p_{i+1}$. This observation helps us to visualize the three line segments.

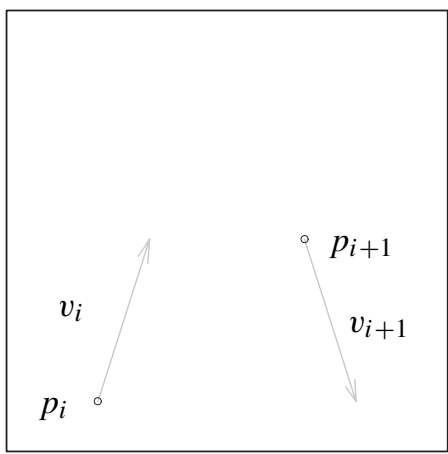

Figure 1: Initial data.

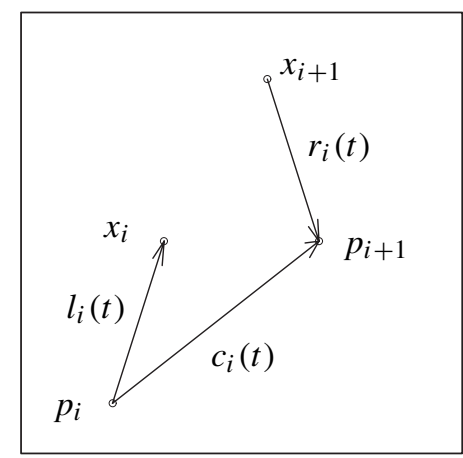

Figure 2: Step 1 - components.

Next, we introduce a smooth real-valued function $\phi:[0,1] \longrightarrow[0,1]$ satisfying

$$
\begin{array}{ll}
\phi(0)=0, & \phi(1)=1, \\
\phi^{(j)}(0)=0, & \phi^{(j)}(1)=0, \quad j=1,2, \ldots, k-1(\text { for } k>1),
\end{array}
$$




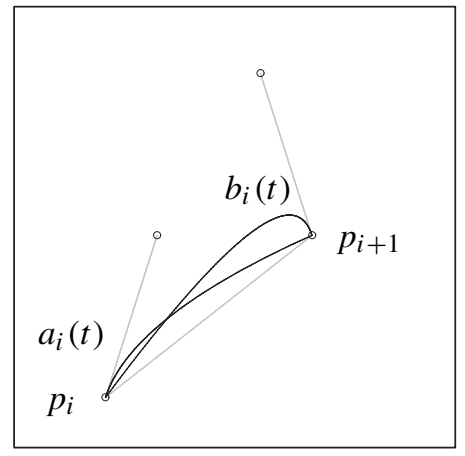

Figure 3: Step 2 - curves $a_{i}$ and $b_{i}$.

and we compute two new curves from convex combinations (using $\phi$ ) of those previously constructed:

$$
\text { Step } 2\left\{\begin{array}{l}
a_{i}(t)=(1-\phi(t)) l_{i}(t)+\phi(t) c_{i}(t) \\
b_{i}(t)=(1-\phi(t)) c_{i}(t)+\phi(t) r_{i}(t)
\end{array}\right.
$$

REMARK 3.2. These new curves are such that $a_{i}(0)=b_{i}(0)=l_{i}(0)=p_{i}, a_{i}(1)=b_{i}(1)=$ $r_{i}(1)=p_{i+1}, \dot{a}_{i}(0)=\dot{l}_{i}(0)=v_{i}$ and $\dot{b}_{i}(1)=\dot{r}_{i}(1)=v_{i+1}$. These boundary conditions do not depend on the choice of the function $\phi$, as long as $\phi$ satisfies conditions (4). For the geometric constructions below, which at this point only help to visualize the steps of the algorithm, we have chosen $\phi(t)=t$. Later, we will explain the relationship between the required degree of smoothness of the spline curve and the choice of the function $\phi$.

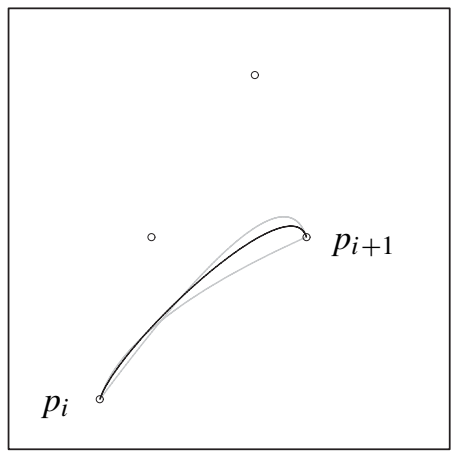

Figure 4: Step 3 - curve $s_{i}$.

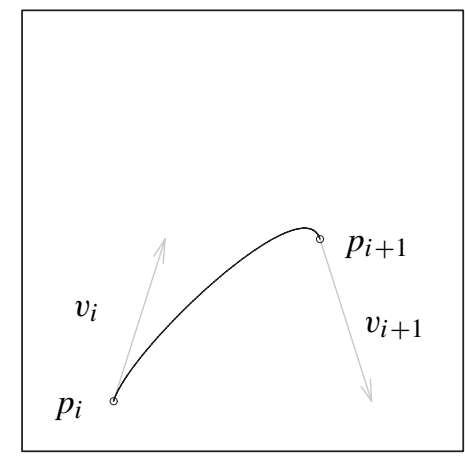

Figure 5: Initial data and final segment.

Finally, we combine $a_{i}$ and $b_{i}$ in a similar way to generate the spline segment:

$$
\underline{\text { Step } 3}\left\{\begin{aligned}
s_{i}(t) & =(1-\phi(t)) a_{i}(t)+\phi(t) b_{i}(t) \\
& =(1-\phi(t))^{2} l_{i}(t)+2 \phi(t)(1-\phi(t)) c_{i}(t)+\phi(t)^{2} r_{i}(t) .
\end{aligned}\right.
$$

The next result presents the main properties of the resulting curve $t \longmapsto s(t)$. 
THEOREM 3.1. If $\phi:[0,1] \longrightarrow[0,1]$ is a smooth function satisfying (4), then the following statments hold.

(i) The spline segment $t \longmapsto s_{i}(t)$ defined by (6), (5) and (2) is smooth and satisfies the following conditions:

$$
\begin{array}{ll}
s_{i}(0)=p_{i}, & s_{i}(1)=p_{i+1}, \\
\dot{s}_{i}(0)=v_{i}, & \dot{s}_{i}(1)=v_{i+1}, \\
s_{i}^{(j)}(0)=0, & s_{i}^{(j)}(1)=0, \quad j=2, \ldots, k .
\end{array}
$$

(ii) The resulting spline curve $t \longmapsto s(t)$ given by

$$
s(t)=s_{i}\left(\frac{t-t_{i}}{t_{i+1}-t_{i}}\right), \quad t \in\left[t_{i}, t_{i+1}\right], i=0,1 \ldots, m-1,
$$

is $C^{k}$-smooth and satisfies the interpolation conditions (1).

Proof. Applying Leibniz's formula for the $j$ th derivative of a product to the formula (6) for the spline segment, we get

$$
s_{i}^{(j)}(t)=\sum_{l=0}^{j}\left(\begin{array}{l}
j \\
l
\end{array}\right)(1-\phi(t))^{(j-l)} a_{i}^{(l)}(t)+\sum_{l=0}^{j}\left(\begin{array}{l}
j \\
l
\end{array}\right) \phi^{(j-l)}(t) b_{i}^{(l)}(t) .
$$

To check all the boundary conditions more easily, we rewrite $s_{i}^{(j)}$ in a more convenient form. Since $(1-\phi(t))^{(j)}=-\phi^{(j)}(t)$ for $j \geqslant 1$, the previous formula can be written as

$$
s_{i}^{(j)}(t)=\sum_{l=0}^{j-1}\left(\begin{array}{l}
j \\
l
\end{array}\right) \phi^{(j-l)}(t)\left(b_{i}(t)-a_{i}(t)\right)^{(l)}+(1-\phi(t)) a_{i}^{(j)}(t)+\phi(t) b_{i}^{(j)}(t) .
$$

Using the same argument to calculate $a_{i}^{(j)}(t)$ and $b_{i}^{(j)}(t)$, we have

$$
\begin{aligned}
a_{i}^{(j)}(t) & =\sum_{l=0}^{j-1}\left(\begin{array}{l}
j \\
l
\end{array}\right) \phi^{(j-l)}(t)\left(c_{i}(t)-l_{i}(t)\right)^{(l)}+(1-\phi(t)) l_{i}^{(j)}(t)+\phi(t) c_{i}^{(j)}(t) ; \\
b_{i}^{(j)}(t) & =\sum_{l=0}^{j-1}\left(\begin{array}{l}
j \\
l
\end{array}\right) \phi^{(j-l)}(t)\left(r_{i}(t)-c_{i}(t)\right)^{(l)}+(1-\phi(t)) c_{i}^{(j)}(t)+\phi(t) r_{i}^{(j)}(t) .
\end{aligned}
$$

Therefore,

$$
\begin{aligned}
s_{i}^{(j)}(t)= & \sum_{l=0}^{j-1}\left(\begin{array}{l}
j \\
l
\end{array}\right) \phi^{(j-l)}(t)\left(b_{i}(t)-a_{i}(t)\right)^{(l)} \\
& +(1-\phi(t)) \sum_{l=0}^{j-1}\left(\begin{array}{l}
j \\
l
\end{array}\right) \phi^{(j-l)}(t)\left(c_{i}(t)-l_{i}(t)\right)^{(l)} \\
& +\phi(t) \sum_{l=0}^{j-1}\left(\begin{array}{l}
j \\
l
\end{array}\right) \phi^{(j-l)}(t)\left(r_{i}(t)-c_{i}(t)\right)^{(l)} \\
& +(1-\phi(t))^{2} l_{i}^{(j)}(t)+2 \phi(t)(1-\phi(t)) c_{i}^{(j)}(t)+\phi(t)^{2} r_{i}^{(j)}(t) .
\end{aligned}
$$


Since $\phi$ satisfies (4) and $c_{i}(0)=l_{i}(0), a_{i}(0)=b_{i}(0), c_{i}(1)=r_{i}(1)$ and $a_{i}(1)=b_{i}(1)$ (see Remarks 3.1 and 3.2), we get

$$
s_{i}^{(j)}(0)=l_{i}^{(j)}(0) \quad \text { and } \quad s_{i}^{(j)}(1)=r_{i}^{(j)}(1),
$$

for all $j=0,1, \ldots, k$. The boundary conditions (7) follow from the properties given in (3). The second part of the theorem is a direct consequence of conditions (7).

REMARK 3.3 (THE COMPLEXITY OF THE ALGORITHM). It is clear that the complexity of our algorithm does not depend on $k$. Indeed, if we want a $C^{k}$-smooth curve (for $k \geqslant 2$ ), our algorithm produces a spline that also satisfies $s^{(j)}\left(t_{i}\right)=0, i=0,1 \ldots, m$, for $j=2, \ldots, k$, in only three steps.

If these conditions were initially prescribed together with conditions (1), the De Casteljau algorithm could also be used to solve the problem. But, as already observed, the complexity of this algorithm increases substantially with $k$, since $2 k+1$ steps are required.

If we choose $\phi(t)=t$, then the spline generated by our algorithm is $C^{1}$-smooth and coincides with that produced by the De Casteljau algorithm. This is not a surprise. In fact, by construction, we know that each segment will be a cubic polynomial in $\mathbb{R}^{n}$ which is uniquely determined, since four data points are given. Each segment of this spline is given by

$$
s_{i}(t)=p_{i}+v_{i} t+\left(3 p_{i+1}-3 p_{i}-2 v_{i}-v_{i+1}\right) t^{2}+\left(2 p_{i}+v_{i}-2 p_{i+1}+v_{i+1}\right) t^{3} .
$$

REMARK 3.4 (THE SMOOTHING FUNCTION). The degree of smoothness of the spline generated by the new algorithm depends only on the choice of a smooth function $\phi:[0,1] \longrightarrow$ $[0,1]$, satisfying (4). This is the reason why we call $\phi$ a 'smoothing function' for the spline curve $s$. One possible choice for the function $\phi$ satisfying all the conditions (4) is the following polynomial function of degree $2 k-1$ :

$$
\phi(t)=\gamma \sum_{l=0}^{k-1} \frac{\alpha_{k+l}}{k+l} t^{k+l}
$$

where

$$
\alpha_{k+l}=(-1)^{l}\left(\begin{array}{c}
k-1 \\
l
\end{array}\right) \quad \text { and } \quad \gamma^{-1}=\sum_{l=0}^{k-1} \frac{\alpha_{k+l}}{k+l} .
$$

The following formulas will soon be useful:

$$
\phi^{(k)}(0)=\gamma(k-1) ! \quad \text { and } \quad \phi^{(k)}(1)=(-1)^{k-1} \gamma(k-1) ! .
$$

The proof relies on the observation that $\phi(t)$ is an anti-derivative of the function

$$
g(t)=\gamma t^{k-1}(1-t)^{k-1}=\gamma \sum_{l=0}^{k-1}(-1)^{l}\left(\begin{array}{c}
k-1 \\
l
\end{array}\right) t^{k-1+l},
$$

which implies that $\phi^{(k)}(t)=g^{(k-1)}(t)$. It is now sufficient to evaluate the derivative of order $k-1$ of function $g$ at $t=0$ and $t=1$. More properties of this smoothing function may be found in [8]. From now on, we will always consider this particular smoothing function. For instance,

- for $k=1$, we have $\phi(t)=t$,

- for $k=2$, we have $\phi(t)=t^{2}(3-2 t)$, and

- for $k=3$, one obtains $\phi(t)=t^{3}\left(10-15 t+6 t^{2}\right)$. 
For the function $\phi$ given by (9), each segment of the generated spline curve is a polynomial curve of degree (at most) $4 k-1$.

We note that the spline $t \longmapsto s(t)$, given by (8), when $\phi$ is as defined in (9), will not be $C^{k+1}$-smooth, except for some degenerate cases. To see this, it is enough to compute $s_{i}^{(k+1)}(1)$ and $s_{i+1}^{(k+1)}(0)$. We have to distinguish between $k=1$ and $k>1$, since for $k=1$ we find the following formulas:

$$
\begin{gathered}
\ddot{s}_{i}(1)=2\left(x_{i}-p_{i+1}\right)+4\left(v_{i+1}-p_{i+1}+p_{i}\right), \\
\ddot{s}_{i+1}(0)=2\left(x_{i+2}-p_{i+1}\right)+4\left(p_{i+2}-p_{i+1}-v_{i+1}\right),
\end{gathered}
$$

and for $k>1$ we have

$$
\begin{gathered}
s_{i}^{(k+1)}(1)=2(k+1) \phi^{(k)}(1)\left(v_{i+1}-p_{i+1}+p_{i}\right), \\
s_{i+1}^{(k+1)}(0)=2(k+1) \phi^{(k)}(0)\left(p_{i+2}-p_{i+1}-v_{i+1}\right) .
\end{gathered}
$$

Now, $\ddot{s}_{i}(1)=\ddot{s}_{i+1}(0)$, for some $i \in\{0,1 \ldots, m-1\}$ if and only if the following holds:

$$
-4 v_{i+1}=\left(x_{i}-x_{i+2}\right)+2\left(p_{i}-p_{i+2}\right) \Longleftrightarrow v_{i}+4 v_{i+1}-v_{i+2}=3\left(p_{i+2}-p_{i}\right),
$$

and for $k>1$, we may use the equalities (10) to conclude that $s_{i}^{(k+1)}(1)=s_{i+1}^{(k+1)}(0)$, for some $i \in\{0,1 \ldots, m-1\}$, if and only if

$$
\begin{cases}2 v_{i+1}=p_{i+2}-p_{i} & \text { if } k \text { is odd } \\ p_{i+1}-p_{i}=p_{i+2}-p_{i+1} & \text { if } k \text { is even. }\end{cases}
$$

REMARK 3.5 (OPTIMAL PROPERTIES). For $k=1$ (that is, $\phi(t)=t$ ), each component of the spline function given by (8) is an $L$-spline (of type I) associated with the differential operator $L=d^{2} / d t^{2}$, the partition $\Delta$ and the incidence vector $\mathrm{Z}=\left(z_{1}, z_{2}, \ldots, z_{m-1}\right)=$ $(2,2, \ldots, 2)$. See [17] for the definition of $L$-splines and their properties.

Consequently, if $\langle.,$.$\rangle represents the Euclidean inner product in \mathbb{R}^{n}$, and $\Omega$ denotes the class of all functions $y:[a, b] \subset \mathbb{R} \longrightarrow \mathbb{R}^{n}$ which are $C^{1}$-smooth in $[a, b]$ and fulfill the interpolation conditions (1), then the spline function given by (8) and corresponding to $\phi(t)=t$ is the solution of the following optimization problem:

$$
\min _{y \in \Omega} \int_{a}^{b}\langle\ddot{y}(t), \ddot{y}(t)\rangle d t .
$$

For $k>1$, the optimal properties of the spline function produced by our algorithm are still under investigation. However, it is interesting to note that the smoothing function $\phi$ defined by (9) is the unique solution of the optimization problem

$$
\min _{f \in C^{k}[0,1]} \int_{0}^{1}\left\langle f^{(k)}(t), f^{(k)}(t)\right\rangle d t,
$$

subject to the following boundary conditions:

$$
\begin{array}{ll}
f(0)=0, & f(1)=1, \\
f^{(j)}(0)=0, & f^{(j)}(1)=0, \quad j=1,2, \ldots, k-1(\text { for } k>1) .
\end{array}
$$

\subsection{Extension to problems with uneven conditions}

The new algorithm is easily adapted to the computation of a $C^{k}$-smooth curve $s$ (where $k \geqslant 1$ ) that fulfills a more challenging set of interpolation conditions of the form

$$
s\left(t_{i}\right)=p_{i}, \quad \dot{s}\left(t_{i}\right)=\dot{p}_{i}, \quad \ddot{s}\left(t_{i}\right)=\ddot{p}_{i}, \quad \ldots \quad s^{\left(k_{i}\right)}\left(t_{i}\right)=p_{i}^{\left(k_{i}\right)},
$$


for the partition $\Delta: a=t_{0}<t_{1}<\cdots<t_{m}=b$ of the time interval $[a, b]$, points $p_{i}$ in $\mathbb{R}^{n}$ and vectors $\dot{p}_{i}, \ddot{p}_{i}, \ldots, p_{i}^{\left(k_{i}\right)}$ tangent to $\mathbb{R}^{n}$ at $p_{i}$, with $1 \leqslant k_{i} \leqslant k$ and $i=0,1, \ldots, m$.

This extension allows uneven prescribed conditions at each instant $t_{i}$, and is of particular importance in many applications. The only changes required are in the left and right components of each segment $s_{i}$. If $k_{i}$ is the number of derivatives prescribed at the initial point $p_{i}$, then the left component for the segment $s_{i}$ is a polynomial of degree $k_{i}$. If $k_{i+1}$ is the number of derivatives prescribed at the end-point $p_{i+1}$, then the right component for the segment $s_{i}$ is a polynomial of degree $k_{i+1}$. Besides these modifications, all remains the same, including the center component. More specifically, to compute the curve $t \longmapsto s_{i}(t)$ that connects the point $p_{i}$ at $t=0$ to $p_{i+1}$ at $t=1$ with prescribed interpolation conditions (11), we use the same control points, which are now written as

$$
x_{i}=p_{i}+\dot{p}_{i}, \quad x_{i+1}=p_{i+1}-\dot{p}_{i+1},
$$

and we define the left and right components to be the Taylor polynomials

$$
l_{i}(t)=\sum_{j=0}^{k_{i}} \frac{p_{i}^{(j)}}{j !} t^{j}, \quad \text { and } \quad r_{i}(t)=\sum_{j=0}^{k_{i+1}} \frac{p_{i+1}^{(j)}}{j !}(t-1)^{j} .
$$

Notice that $l_{i}$ and $r_{i}$ are such that

$$
\begin{aligned}
& l_{i}(0)=p_{i}, \\
& \dot{l}_{i}(0)=\dot{p}_{i} \text {, } \\
& r_{i}(1)=p_{i+1}, \\
& \dot{r}_{i}(1)=\dot{p}_{i+1} \text {, } \\
& l_{i}^{\left(k_{i}\right)}(0)=p_{i}^{\left(k_{i}\right)}, \\
& r_{i}^{\left(k_{i+1}\right)}(1)=p_{i+1}^{\left(k_{i+1}\right)}, \\
& l_{i}^{(j)}(0)=0, \quad j>k_{i}, \\
& r_{i}^{(j)}(1)=0, \quad j>k_{i+1} .
\end{aligned}
$$

As before, we use a smoothing function $\phi$ satisfying (4), and we compute $a_{i}, b_{i}$ and $s_{i}$ as described on page 255. The next result, similar to Theorem 3.1, follows immediately.

COROllary 3.1. If $\phi:[0,1] \longrightarrow[0,1]$ is a smooth function satisfying (4), then the following statements hold.

(i) The spline segment $t \longmapsto s_{i}(t)$ defined by

$$
s_{i}(t)=(1-\phi(t))^{2} l_{i}(t)+2 \phi(t)(1-\phi(t)) c_{i}(t)+\phi(t)^{2} r_{i}(t)
$$

where $l_{i}$ and $r_{i}$ are given by (12) and $c_{i}$ is given by (2), satisfies the following conditions:

$$
\begin{array}{rlrl}
s_{i}(0) & =p_{i}, & s_{i}(1) & =p_{i+1}, \\
\dot{s}_{i}(0) & =\dot{p}_{i}, & \dot{s}_{i}(1) & =\dot{p}_{i+1}, \\
& \vdots & \vdots \\
s_{i}^{\left(k_{i}\right)}(0) 7 & =p_{i}^{\left(k_{i}\right)}, & s_{i}^{\left(k_{i+1}\right)}(1) & =p_{i+1}^{\left(k_{i+1}\right)}, \\
s_{i}^{(j)}(0) 7 & =0, \quad j=k_{i}+1, \ldots, k, \quad s_{i}^{(j)}(1) & =0, \quad j=k_{i+1}+1, \ldots, k .
\end{array}
$$

(ii) the resulting spline curve $t \longmapsto s(t)$ given by

$$
s(t)=s_{i}\left(\frac{t-t_{i}}{t_{i+1}-t_{i}}\right), \quad t \in\left[t_{i}, t_{i+1}\right], i=0,1 \ldots, m-1,
$$

is $C^{k}$-smooth and satisfies the set of interpolation conditions (11). 


\section{The new algorithm on complete Riemannian manifolds}

In this section we combine the ideas just developed to implement the new algorithm in Euclidean spaces, with those used to generalize the De Casteljau algorithm to complete Riemannian manifolds (see details in [3]). In this section we consider the case when only points and first derivatives are prescribed, since for higher derivatives the implementation of the algorithm requires the analogues of higher-order polynomials on manifolds. Now, geodesic arcs play the role of straight-line segments; consequently, the algorithm is generally applicable as long as the computation of geodesics is tractable. When the manifold is a compact and connected Lie group $G$, equipped with the left- and right-invariant Riemannian metric, geodesics are easily expressed in terms of one-parameter subgroups. When the manifold is a unit sphere, equipped with the Riemannian metric induced by the Euclidean metric in the embedding space, geodesics are just great circles. We next describe the new algorithm for these two special Riemannian manifolds.

\subsection{The new algorithm on Lie groups}

In this section, $G$ is a connected and compact Lie group, equipped with the unique rightand left-invariant Riemannian metric, and $\mathcal{L}$ denotes its Lie algebra. Elements in $\mathcal{L}$ will be represented by capital letters.

Similarly to the Euclidean case, the construction of the spline curve that solves the initial problem is local, so the details will be presented only for the construction of the spline segment $t \longmapsto s_{i}(t)$ that joins two given points in $G, p_{i}$ (at $\left.t=0\right)$ and $p_{i+1}($ at $t=1)$, with prescribed velocities $v_{i}=V_{i} p_{i}$ and $v_{i+1}=V_{i+1} p_{i+1}$, where $V_{i}$ and $V_{i+1}$ belong to the Lie algebra of $G$. The smoothing function is the same as in the Euclidean case.

We now describe the three basic steps that are used to obtain the required spline segment $t \longmapsto s_{i}(t)$.

Step 1: We first construct the geodesic segments which are the left, center and right components, and are defined by:

$$
\begin{aligned}
l_{i}(t) & =e^{t V_{i}} p_{i}, \\
c_{i}(t) & =e^{t W_{i}} p_{i}, \quad \text { where } W_{i}=\log \left(p_{i+1} p_{i}^{-1}\right), \\
r_{i}(t) & =e^{(t-1) V_{i+1}} p_{i+1} .
\end{aligned}
$$

The following boundary conditions are easily checked:

$$
\begin{array}{ll}
l_{i}(0)=p_{i}, & l_{i}(1)=e^{V_{i}} p_{i}, \\
\dot{l}_{i}(0)=V_{i} p_{i}, & \dot{l}_{i}(1)=V_{i} e^{V_{i}} p_{i}, \\
c_{i}(0)=p_{i}, & c_{i}(1)=p_{i+1} \\
\dot{c}_{i}(0)=W_{i} p_{i}, & \dot{c}_{i}(1)=W_{i} e^{W_{i}} p_{i}, \\
r_{i}(0)=e^{-V_{i+1}} p_{i+1}, & r_{i}(1)=p_{i+1}, \\
\dot{r}_{i}(0)=V_{i+1} e^{-V_{i+1}} p_{i+1}, & \dot{r}_{i}(1)=V_{i+1} p_{i+1} .
\end{array}
$$

Step 2: Now we define $t \longmapsto a_{i}(t)$ and $t \longmapsto b_{i}(t)$ by:

$$
\begin{array}{ll}
a_{i}(t)=e^{\phi(t) A_{i}(t)} l_{i}(t), & \text { where } A_{i}(t)=\log \left(c_{i}(t) l_{i}^{-1}(t)\right), \\
b_{i}(t)=e^{\phi(t) B_{i}(t)} c_{i}(t), & \text { where } B_{i}(t)=\log \left(r_{i}(t) c_{i}^{-1}(t)\right) .
\end{array}
$$


The following alternative formulas for these two curves will simplify checking the boundary conditions of the spline curve at $t=1$ :

$$
\begin{aligned}
& a_{i}(t)=e^{-(1-\phi(t)) A_{i}(t)} c_{i}(t) ; \\
& b_{i}(t)=e^{-(1-\phi(t)) B_{i}(t)} r_{i}(t) .
\end{aligned}
$$

Indeed,

$$
\begin{aligned}
e^{-(1-\phi(t)) A_{i}(t)} c_{i}(t) & =e^{\phi(t) A_{i}(t)} e^{-A_{i}(t)} c_{i}(t) \\
& =e^{\phi(t) A_{i}(t)} e^{-\log \left(c_{i}(t) l_{i}^{-1}(t)\right)} c_{i}(t) \\
& =e^{\phi(t) A_{i}(t)} l_{i}(t) c_{i}^{-1}(t) c_{i}(t) \\
& =e^{\phi(t) A_{i}(t)} l_{i}(t) \\
& =a_{i}(t)
\end{aligned}
$$

and similarly for $b_{i}$.

Now, using the conditions (4) for the smoothing function $\phi$ and the boundary conditions (13), we obtain from (14) and (15) the following:

$$
\begin{array}{ll}
a_{i}(0)=b_{i}(0)=p_{i}, & a_{i}(1)=b_{i}(1)=p_{i+1}, \\
\dot{a}_{i}(0)=V_{i} p_{i}, & \dot{a}_{i}(1)=W_{i} e^{W_{i}} p_{i}, \\
\dot{b}_{i}(0)=W_{i} p_{i}, & \dot{b}_{i}(1)=V_{i+1} p_{i+1} .
\end{array}
$$

Step 3: Finally, we define the spline segment $t \longmapsto s_{i}(t)$ by

$$
s_{i}(t)=e^{\phi(t) S_{i}(t)} a_{i}(t), \quad \text { where } S_{i}(t)=\log \left(b_{i}(t) a_{i}^{-1}(t)\right),
$$

or, similarly,

$$
s_{i}(t)=e^{\phi(t) S_{i}(t)} e^{\phi(t) A_{i}(t)} e^{t V_{i}} p_{i}
$$

THEOREM 4.1. If $\phi:[0,1] \longrightarrow[0,1]$ is a smooth function satisfying (4), then the curve $t \longmapsto s_{i}(t)$ defined by (16) satisfies the following boundary conditions:

$$
\begin{aligned}
s_{i}(0) & =p_{i}, & & s_{i}(1)=p_{i+1}, \\
\dot{s}_{i}(0) & =V_{i} p_{i}, & & \dot{s}_{i}(1)=V_{i+1} p_{i+1} .
\end{aligned}
$$

Proof. We first derive, from (16), an expression for the first derivative of $s_{i}$. Using the Campbell-Hausdorff formula

$$
e^{A(t)} B(t) e^{-A(t)}=e^{\mathrm{ad} A(t)} B(t)=\sum_{j=0}^{+\infty} \mathrm{ad}^{j} A(t)(B(t)),
$$

where 'ad' denotes the adjoint operator on $\mathcal{L}$ defined by ad $A(B)=[A, B]$, and using also the following formula for the derivative of the exponential, which may be found in [16]:

$$
\frac{d}{d t}\left(e^{A(t)}\right)=\Omega_{A}^{L}(t) e^{A(t)}, \quad \text { where } \Omega_{A}^{L}(t)=\int_{0}^{1} e^{u \text { ad } A(t)} \dot{A}(t) d u,
$$


we obtain

$$
\begin{aligned}
\dot{s}_{i}(t)=\Omega_{\phi S_{i}}^{L}(t) s_{i}(t) & +e^{\phi(t) S_{i}(t)} \Omega_{\phi A_{i}}^{L}(t) e^{\phi(t) A_{i}(t)} e^{t V_{i}} p_{i} \\
& +e^{\phi(t) S_{i}(t)} e^{\phi(t) A_{i}(t)} V_{i} e^{t V_{i}} p_{i} \\
=\Omega_{\phi S_{i}}^{L}(t) s_{i}(t) & +e^{\phi(t) S_{i}(t)} \Omega_{\phi A_{i}}^{L}(t) e^{-\phi(t) S_{i}(t)} s_{i}(t) \\
& +e^{\phi(t) S_{i}(t)} e^{\phi(t) A_{i}(t)} V_{i} e^{-\phi(t) A_{i}(t)} e^{-\phi(t) S_{i}(t)} s_{i}(t) \\
= & \left(\Omega_{\phi S_{i}}^{L}(t)+e^{\phi(t) \text { ad } S_{i}(t)} \Omega_{\phi A_{i}}^{L}(t)+e^{\phi(t) \operatorname{ad} S_{i}(t)} e^{\phi(t) \text { ad } A_{i}(t)} V_{i}\right) s_{i}(t) .
\end{aligned}
$$

The initial conditions $s_{i}(0)=p_{i}$ and $\dot{s}_{i}(0)=V_{i} p_{i}$, follow easily from (16) and (17), if we take into consideration that $\phi(0)=0$. To prove that the conditions at $t=1$ are also satisfied, we rewrite the expression for $s_{i}$ given in (16), similarly to what has been done for the curves obtained in Step 2, to obtain

$$
s_{i}(t)=e^{-(1-\phi(t)) S_{i}(t)} e^{-(1-\phi(t)) B_{i}(t)} e^{-(1-t) V_{i+1}} p_{i+1} .
$$

Consequently, an alternative expression for the first derivative is now as follows:

$$
\dot{s}_{i}(t)=\left(\Omega_{-\psi S_{i}}^{L}(t)+e^{-\psi(t) \text { ad } S_{i}(t)} \Omega_{-\psi B_{i}}^{L}(t)+e^{-\psi(t) \operatorname{ad} S_{i}(t)} e^{-\psi(t) \operatorname{ad} B_{i}(t)} V_{i+1}\right) s_{i}(t),
$$

where $\psi(t)=1-\phi(t)$, so that $\psi(1)=0$. The final conditions, $s_{i}(1)=p_{i+1}$ and $\dot{s}_{i}(1)=$ $V_{i+1} p_{i+1}$, follow easily from (18) and (19).

To show that when piecing together the spline segments, the resulting spline curve is $C^{k}$-smooth, one needs to derive higher-order covariant derivatives. The covariant derivative of a vector field along a curve in $G$ (a manifold imbedded in some high-dimensional Euclidean space $\mathbb{R}^{n}$ ) may be viewed as a new vector field along that curve, which results from differentiating as a vector field along a curve in $\mathbb{R}^{n}$ and then projecting it, at each point, onto the tangent space to $G$ at that point. The details are rather technical, and will be omitted here. Nevertheless, when $G$ is the Lie group of rotations $\mathrm{SO}(n)$, with Lie algebra so $(n)$ consisting of all $n \times n$ skew-symmetric matrices, the Riemannian metric is defined by $\langle A, B\rangle=\operatorname{trace}\left(A^{T} B\right), A, B \in \operatorname{so}(n)$, and the tangent space at a point $p \in \operatorname{SO}(n)$ and its orthogonal complement with respect to $\langle.,$.$\rangle are, respectively,$

$$
T_{p} \operatorname{SO}(n)=\{A p: A \in \operatorname{so}(n)\} \quad \text { and } \quad T_{p}^{\perp} \operatorname{SO}(n)=\{\operatorname{Sp}: S \in s(n)\},
$$

where $s(n)$ is the set of all $n \times n$ symmetric matrices.

In this case, and after many calculations that are omitted here, we reach the final conclusion, which is a generalization to the Lie group $\mathrm{SO}(n)$ of Theorem 3.1.

THEOREM 4.2. If $\phi:[0,1] \longrightarrow[0,1]$ is a smooth function satisfying (4), then the following statements hold.

(i) The spline segment $t \longmapsto s_{i}(t) \in \mathrm{SO}(n)$ defined by (16) satisfies the following boundary conditions

$$
\begin{array}{ll}
s_{i}(0)=p_{i}, & s_{i}(1)=p_{i+1}, \\
\dot{s}_{i}(0)=V_{i} p_{i}, & \dot{s}_{i}(1)=V_{i+1} p_{i+1}, \\
\frac{D^{j} \dot{s}_{i}}{d t^{j}}(0)=0, & \frac{D^{j} \dot{s}_{i}}{d t^{j}}(1)=0, \quad j=1, \ldots, k-1 .
\end{array}
$$


(ii) The resulting spline curve $t \longmapsto s(t) \in \mathrm{SO}(n)$ given by

$$
s(t)=s_{i}\left(\frac{t-t_{i}}{t_{i+1}-t_{i}}\right), \quad t \in\left[t_{i}, t_{i+1}\right], i=0,1 \ldots, m-1,
$$

is $C^{k}$-smooth and satisfies the interpolation conditions (1).

\subsection{The new algorithm on spheres}

Here we describe the geometric algorithm to construct a $C^{2}$-smooth spline curve interpolating a given set of points on the unit sphere $S^{n}$, with prescribed velocities through those points. We consider $S^{n}$ equipped with the Riemannian metric induced by the Euclidean metric in the embedding space $\mathbb{R}^{n+1}$. As before, we start with the construction of a natural spline segment between two points. This means that the second covariant derivatives are zero at the boundary, which consequently guarantees the $C^{2}$ smoothness of the interpolating curve, as soon as all the spline segments are glued together. In order to describe the geometric algorithm that generates the natural spline segment $t \in[0,1] \longmapsto s_{i}(t) \in S^{n}$ joining two given points $p_{i}$ and $p_{i+1}$, with prescribed initial and final velocities, we first recall some properties of geodesics on spheres.

Given a point $x_{0} \in S^{n}$ and a vector $v_{0}$ tangent to the sphere at $x_{0}$, there exists a unique geodesic $t \longmapsto x(t)$ that passes through $x_{0}$ at time $\tau$, with velocity $v_{0}$ :

$$
x(t)=\cos \left((t-\tau)\left\|v_{0}\right\|\right) x_{0}+\sin \left((t-\tau)\left\|v_{0}\right\|\right) \hat{v}_{0},
$$

where $\hat{v}_{0}=v_{0} /\left\|v_{0}\right\|$.

Also, given two (not antipodal) points $y_{0}, y_{1} \in S^{n}$, the geodesic $\operatorname{arc} t \longmapsto y(t)$ which joins $y_{0}($ at $t=0)$ to $y_{1}$ (at $\left.t=1\right)$ is given by:

$$
y(t)=\frac{\sin \left((1-t) \theta_{y_{0}, y_{1}}\right)}{\sin \theta_{y_{0}, y_{1}}} y_{0}+\frac{\sin \left(t \theta_{y_{0}, y_{1}}\right)}{\sin \theta_{y_{0}, y_{1}}} y_{1},
$$

where $\theta_{y_{0}, y_{1}}=\cos ^{-1}\left(y_{0}^{T} y_{1}\right)$ is the angle between the vectors $y_{0}$ and $y_{1}$.

The formula (20) is used to generate the left component $t \longmapsto l_{i}(t)$ and the right component $t \longmapsto r_{i}(t)$ of the natural spline segment $t \longmapsto s_{i}(t)$ that joins the points $p_{i}$ (at $t=0$ ) to $p_{i+1}$ (at $t=1$ ) with prescribed initial and final velocity $v_{i}$ and $v_{i+1}$ respectively. The formula (21) is used to generate the center component $t \longmapsto c_{i}(t)$ and the intermediate curves in the algorithm below. Taking into consideration that the left component is a geodesic satisfying the same conditions as the spline segment at $t=0$, that the right component is a geodesic satisfying the same conditions as the spline segment at $t=1$, and that the center component joins the points $p_{i}($ at $t=0)$ and $p_{i+1}($ at $t=1)$, the algorithm is performed in the following three steps.

Step 1: Construct the left, center and right components, defined by:

$$
\begin{aligned}
& l_{i}(t)=\cos \left(t\left\|v_{i}\right\|\right) p_{i}+\sin \left(t\left\|v_{i}\right\|\right) \hat{v}_{i}, \\
& c_{i}(t)=\frac{\sin \left((1-t) \theta_{p_{i}, p_{i+1}}\right)}{\sin \theta_{p_{i}, p_{i+1}}} p_{i}+\frac{\sin \left(t \theta_{p_{i}, p_{i+1}}\right)}{\sin \theta_{p_{i}, p_{i+1}}} p_{i+1}, \\
& r_{i}(t)=\cos \left((t-1)\left\|v_{i+1}\right\|\right) p_{i+1}+\sin \left((t-1)\left\|v_{i+1}\right\|\right) \hat{v}_{i+1} .
\end{aligned}
$$


Step 2: Now define $t \longmapsto a_{i}(t)$ and $t \longmapsto b_{i}(t)$ using convex combinations (parameterized by the smoothing function $\phi$ ) of the geodesics in the previous step:

$$
\begin{aligned}
& a_{i}(t)=\frac{\sin \left((1-\phi(t)) \theta_{l_{i}(t), c_{i}(t)}\right)}{\sin \theta_{l_{i}(t), c_{i}(t)}} l_{i}(t)+\frac{\sin \left(\phi(t) \theta_{l_{i}(t), c_{i}(t)}\right)}{\sin \theta_{l_{i}(t), c_{i}(t)}} c_{i}(t), \\
& b_{i}(t)=\frac{\sin \left((1-\phi(t)) \theta_{c_{i}(t), r_{i}(t)}\right)}{\sin \theta_{c_{i}(t), r_{i}(t)}} c_{i}(t)+\frac{\sin \left(\phi(t) \theta_{c_{i}(t), r_{i}(t)}\right)}{\sin \theta_{c_{i}(t), r_{i}(t)}} r_{i}(t) .
\end{aligned}
$$

Step 3: Finally, we obtain the required curve:

$$
s_{i}(t)=\frac{\sin \left((1-\phi(t)) \theta_{a_{i}(t), b_{i}(t)}\right)}{\sin \theta_{a_{i}(t), b_{i}(t)}} a_{i}(t)+\frac{\sin \left(\phi(t) \theta_{a_{i}(t), b_{i}(t)}\right)}{\sin \theta_{a_{i}(t), b_{i}(t)}} b_{i}(t) .
$$

In order to check that the last curve satisfies all the requirements, it is enough to compute the first and second derivatives, and evaluate them at $t=0$ and $t=1$. This is a tedious calculation that we omit here, but it can be easily checked using the following boundary conditions for the curves constructed in this algorithm and for the smoothing function $\phi$ :

$$
\begin{array}{ll}
s_{i}(0)=a_{i}(0)=l_{i}(0)=p_{i}, & s_{i}(1)=b_{i}(1)=r_{i}(1)=p_{i+1}, \\
\dot{s}_{i}(0)=\dot{a}_{i}(0)=\dot{l}_{i}(0)=v_{i}, & \dot{s}_{i}(1)=\dot{b}_{i}(1)=\dot{r}_{i}(1)=v_{i+1}, \\
\ddot{s}_{i}(0)=\ddot{a}_{i}(0)=\ddot{l}_{i}(0)=-\left\|v_{i}\right\|^{2} p_{i}, & \ddot{s}_{i}(1)=\ddot{b}_{i}(1)=\ddot{r}_{i}(1)=-\left\|v_{i+1}\right\|^{2} p_{i+1} .
\end{array}
$$

The last two expressions imply that $\ddot{s}_{i}(0)$ and $\ddot{s}_{i}(1)$ are orthogonal to $S^{n}$ at $p_{i}$ and $p_{i+1}$ respectively, so that the second covariant acceleration vanishes at the boundary points. This observation, together with the other boundary conditions (23), is enough to conclude the following theorem.

THEOREM 4.3. If $\phi:[0,1] \longrightarrow[0,1]$ is any smooth function satisfying $(4)$, then the spline segment $t \longmapsto s_{i}(t)$ defined by (22) satisfies the following boundary conditions:

$$
\begin{array}{ll}
s_{i}(0)=p_{i}, & s_{i}(1)=p_{i+1}, \\
\dot{s}_{i}(0)=v_{i}, & \dot{s}_{i}(1)=v_{i+1}, \\
\frac{D^{2} \dot{s}_{i}}{d t^{2}}(0)=0, & \frac{D^{2} \dot{s}_{i}}{d t^{2}}(1)=0 .
\end{array}
$$

Figure 6 illustrates the result of applying the algorithm above, to generate a natural spline segment satisfying the following data:

$$
\begin{aligned}
p_{i} & =(\sqrt{3} / 2,-\sqrt{3} / 4,1 / 4), \\
p_{i+1} & =(1 / 2, \sqrt{3} / 4,-3 / 4), \\
v_{i} & =(1 / 2, \sqrt{3}, 3-\sqrt{3}), \\
v_{i+1} & =(1 / 10,9 / 5,(9 \sqrt{3}-1) / 15) .
\end{aligned}
$$




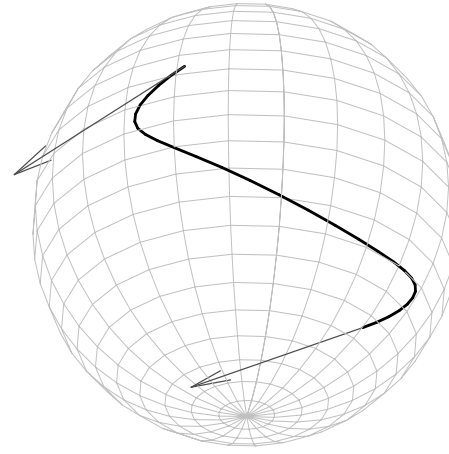

Figure 6: Segment $s_{i}$.

\section{References}

1. A. Barr, B. Currin, S. Gabriel and J. Hughes, 'Smooth interpolation of orientations with angular velocity constraints using quaternions', Proc. Computer Graphics (SIGRAPH'92), July 1992 (ed. B. Currin, S. Gabriel and J. F. Hughes; ACM SIGGRAPH Publications, Chicago, IL, 1992) 313-320.252

2. Снао-Снi Chen, 'Interpolation of orientation matrices using sphere splines in computer animation', MSc thesis, Arizona State University (1990). 252

3. P. Crouch, G. Kun and F. Silva Leite, 'De Casteljau algorithm for cubic polynomials on the rotation group', Proc. Second Portuguese Conference on Automatic Control, Porto, Portugal, 11-13 September 1996 (ed. J. Martins de Carvalho, et al., Portuguese Association of Automatic Control (APCA), Porto, Portugal, 1996) 547-552.252, 260

4. P. Crouch, G. Kun and F. Silva Leite, 'The De Casteljau algorithm on Lie groups and spheres', J. Dynam. Control Systems 5 (1999) 397-429.252, 253

5. P. De Casteluau, 'Outillages méthodes calcule', Technical report, Citroen, A., Paris (1959). 251

6. Gerald FARIn, Curves and surfaces for computer aided geometric design, 3rd edn, Computer Science and Scientific Computing (Academic Press, Boston, MA, 1993). 251,253

7. Q. J. GE and B. Ravani, 'Computer aided geometric design of motion interpolants', J. Mech. Design 118 (1994) 756-762.252

8. J. Jakubiak, F. Silva Leite and R. C. Rodrigues, 'A two-step algorithm of smooth spline generation on Riemannian manifolds', J. Comput. Appl. Math., to appear. 252, 257

9. M. J. KIm, M. S. KIM and S. Y. SHIN, 'A general construction scheme for unit quaternion curves with simple high order derivatives', Proc. Computer Graphics, (SIGRAPH'95), Los Angeles, 1995 (ed. Robert Cook; ACM SIGGRAPH Publications, Los Angeles, CA, 1995) 369-376.252

10. M. S. NAgY and T. P. Vendel, 'Generating curves and swept surfaces by blended circles', Computer Aided Geometric Design 17 (2000) 197-206. 252 
11. G. NiELson, 'Smooth interpolation of orientations', Models and techniques in computer animation (ed. N. Magnat Thalmann and D. Thalmann; Springer, Tokyo, 1994) $75-93.252$

12. G. NiELSON and R. HeIlAnd, 'Animated rotations using quaternions and splines on a 4D sphere', Program. Comput. Software 18 (4) (1992) 145-154. 252

13. F. C. PARK and B. Ravani, 'Bézier curves on Riemannian manifolds and Lie groups with kinematic applications', ASME J. Mech. Design 117 (1995) 36-40.252, 253

14. R. C. Rodrigues and F. Silva Leite, 'A new geometric algorithm to generate spline curves', Proc. Sixteenth International Symposium on Mathematical Theory of Networks and Systems (MTNS2004), Katholieke Universiteit Leuven, Belgium, 5-9 July 2004, CD-ROM paper 311.PDF. 252

15. R. C. Rodrigues, F. Silva Leite and S. Rosa, 'On the generation of a trigonometric interpolating curve in $\mathbb{R}^{3}$, Proc. 11th International Conference on Advanced Robotics, ICAR2003, Coimbra, Portugal, 30 June - 3 July 2003, CD-ROM paper N1629.PDF. 252

16. D. H. SATtinger and O. L. Weaver, Lie groups and algebras with applications to physics, geometry, and mechanics, Appl. Math. Sci. 61 (Springer, New York, 1993). 261

17. M. H. Schultz and R. S. VARgA, 'L-splines', Numer. Math 10 (1967) 345-369.258

18. K. ShOEMAKe, 'Animating rotation with quaternion curves', ACM SIGGRAPH'85 19 (1985) 245-254.251, 252

Rui C. Rodrigues ruicreisec.pt

http://www.isec.pt/ ruicr/

Departamento de Física e Matemática

Instituto Superior de Engenharia de Coimbra

Rua Pedro Nunes

3030-199 Coimbra

Portugal

F. Silva Leite fleite@mat.uc.pt

http://www.mat.uc.pt/ fleite/

Departamento de Matemática

Universidade de Coimbra

3001-454 Coimbra

Portugal

Janusz Jakubiak Janusz.Jakubiak@pwr.wroc.pl

Institute of Engineering Cybernetics

Wroclaw University of Technology

ul. Janiszewskiego 11/17

50-370 Wroclaw

Poland 